\title{
Hypoxia-Responsive Nanoreactors to Amplify Oxidative Damage Through Multiple Cascaded Pathways for Promoting Photodynamic/Ferroptosis- Induced ROS Generation and Simultaneously Disrupting Tumor Antioxidant Defense System
}

\section{Xiaoyan Wang}

Fujian Agriculture and Forestry University

\section{Ming Wu}

Mengchao Hepatobiliary Hospital of Fujian Medical University

Xiaolong Zhang

Mengchao Hepatobiliary Hospital of Fujian Medical University

\section{Feida Li}

Fujian Agriculture and Forestry University

\section{Yongyi Zeng}

Mengchao Hepatobiliary Hospital of Fujian Medical University

Xinyi Lin

Mengchao Hepatobiliary Hospital of Fujian Medical University https://orcid.org/0000-0002-9075-5364 Xiaolong Liu ( $\square$ xiaoloong.liu@gmail.com)

Mengchao Hepatobiliary Hospital of Fujian Medical University https://orcid.org/0000-0002-3096-4981 Jingfeng Liu

Mengchao Hepatobiliary Hospital of Fujian Medical University

\section{Research}

Keywords: Oxidation treatment, Photodynamic therapy (PDT), Ferroptosis, Hypoxia-responsive, Proteinbased nanoparticle

Posted Date: May 18th, 2021

DOI: https://doi.org/10.21203/rs.3.rs-523891/v1

License: (c) (i) This work is licensed under a Creative Commons Attribution 4.0 International License.

Read Full License 
Version of Record: A version of this preprint was published at Journal of Nanobiotechnology on July 8th, 2021. See the published version at https://doi.org/10.1186/s12951-021-00952-y. 


\section{Abstract}

Background: Photodynamic therapy (PDT), a typical reactive oxygen species (ROS)-dependent treatment with high controllability, has emerged as an alternative cancer therapy modality but its therapeutic efficacy is still unsatisfactory due to the limited light penetration and constant oxygen consumption. With the development of another ROS-dependent paradigm ferroptosis, several efforts have been made to conquer the poor efficacy by combining these two approaches; however the biocompatibility, tumortargeting capacity and clinical translation prospect of current systems still exist great concerns. Herein, a novel hypoxia-responsive nanoreactor BCFe@SRF with sorafenib (SRF) loaded inside, constructed by covalently connecting chlorin e6 conjugated bovine serum albumin (BSA-Ce6) and ferritin through azobenzene (Azo) linker, were prepared to offer unmatched opportunities for high-efficient PDT and ferroptosis synergistic therapy.

Results: The designed BCFe@SRF exhibited appropriate size distribution, stable dispersity, excellent ROS generation property, controllable drug release capacity, tumor accumulation ability, and outstanding biocompatibility. Importantly, the BCFe@SRF could be degraded under hypoxia environment to release BSA-Ce6 for laser-triggered PDT, ferritin for iron-catalyzed Fenton reaction and SRF for tumor antioxidative defense disruption. Meanwhile, besides PDT effects, it was found that BCFe@SRF mediated treatment upon laser irradiation in hypoxic environment not only could accelerate lipid peroxidation (LPO) generation but also could deplete intracellular glutathione (GSH) and glutathione peroxidase (GPX4) expression, which was believed as three symbolic events during ferroptosis. All in all, the BCFe@SRF nanoreactor, employing multiple cascaded pathways to promote intracellular ROS accumulation, presented remarkably outstanding antitumor effects both in vitro and in vivo.

Conclusion: BCFe@SRF could serve as a promising candidate for synergistic PDT and ferroptosis therapy, which is applicable to boost oxidative damage within tumor site and will be informative to future design of ROS-dependent therapeutic nanoplatforms.

\section{Background}

Reactive oxygen species (ROS)-dependent treatment, as an alternative cancer therapeutic paradigm, has recently gained tremendous attentions [1, 2]. ROS is a family of incomplete reductive molecular forms of oxygen including singlet oxygen $\left({ }^{1} \mathrm{O}_{2}\right)$, hydroxyl radical $(\cdot \mathrm{OH})$, superoxide anion $\left(\mathrm{O}_{2}{ }^{\cdot-}\right)$, hydrogen peroxide $\left(\mathrm{H}_{2} \mathrm{O}_{2}\right)$, etc., which cause oxidative damage to cells through destroying lipid, protein and DNA structures $[1,3,4]$. Photodynamic therapy (PDT), a typical oxidative therapeutic modality, employs photo-activated photosensitizers (PSs) to transfer the absorbing laser energy to surrounding oxygen molecules and subsequently generate high levels of ${ }^{1} \mathrm{O}_{2}$ to kill cancer cells [5, 6]. As is well known, PDT can be operated within a controllably restricted area so that it presents enhanced selectivity and limited side effects [7, 8]. However, the low tumor accumulation of PSs after intravenous injection, limited light penetration and the constant oxygen consumption during PDT make it difficult to produce enough ROS in vivo [7-9]. In addition, cancer cells have oxidation defense systems, expressing high concentration of intracellular 
antioxidant glutathione (GSH), which would hamper the performance of ROS and significantly compromise PDT efficacy $[10,11]$. Conceivably, boosting ROS production within tumor sites and simultaneously disrupting the oxidation defense system of tumor cells should be considered as a promising strategy to improve the antitumor efficiency.

Chemodynamic therapy (CDT), an emerging oxidation therapy depending on the so-called Fenton-type reaction, utilizes transition metals (e.g. $\mathrm{Fe}, \mathrm{Mn}, \mathrm{Cu}, \mathrm{Ni}, \mathrm{Co}$ ) as catalysts to convert the mild oxidant $\mathrm{H}_{2} \mathrm{O}_{2}$ into highly toxic $\cdot \mathrm{OH}$ to kill cancer cells [12-19]. CDT generates ROS originating from $\mathrm{H}_{2} \mathrm{O}_{2}$ which is overexpressed in the tumor microenvironment (TME) instead of oxygen, so that it is not limited by hypoxia as well as light penetration and it also shows tumor-site-specific ROS generation property [20]. Therefore, CDT has enormous potential to be combined with PDT to amplify the oxidative stress within tumor sites. As reported, Fe is the most suitable Fenton-type reaction catalyst for therapeutic purpose [21], which could produce $\cdot \mathrm{OH}$ and then lipid peroxidation (LPO) in tumor sites with a high level of $\mathrm{H}_{2} \mathrm{O}_{2}$. Different from apoptotic cell death which is believed as the major mechanism of PDT [11]. This irondependent LPO-associated oxidative damage causes a non-apoptotic form of regulated cell death type that is newly defined as ferroptosis [21-25]. Recently discovered ferroptosis-inducing agents mainly include iron-based materials (e.g. inorganic iron-involved nanoparticles (NPs), Fe-metal-organic frameworks (Fe-MOFs), iron storage or transport proteins) [1, 3, 20, 24-26] and certain small molecule drugs (e.g. sorafenib (SRF), erastin, sulfasalazine, artemisinin) [23, 27-29], the former accelerating ironcatalyzed ROS production and the latter inhibiting GSH biosynthesis and then depleting glutathione peroxidase 4 (GPX4, the lipid repair enzyme) generation, eventually leading to large amounts of intracellular LPO accumulation. Owing to both the homologous ROS production modalities with tumor specificity, integrating PDT and ferroptosis therapy may provide a huge opportunity to amplify oxidative stress to relatively more thoroughly eliminate tumor cells.

Nanoparticle platforms offer an unmatched potential for tumor-targeting simultaneous delivery of PS, exogenous iron and small GSH/GPX4 depletion molecule [30-32]. Recently, several Fe-MOFs or Fe-based inorganic nanoparticles have been emerged for PDT and ferroptosis combining therapy, achieving significantly enhanced therapeutic outcomes in tumor oxidation treatments [11, 20,26-29], however, the biocompatibility, tumor-targeting capacity and clinical translation prospect of which still exist great concerns. Additionally, these nanosystems utilized the acid condition or high concentration of matrix metalloproteinase (MMP) of TME to trigger cargo release, but these differentiations between normal tissues and tumor tissues are not significant enough and easily be affected by the tumor heterogeneity [33-35]. Therefore, novel strategies based on more biocompatible nanoplatforms with more specific responsiveness property are needed to be developed for PDT and ferroptosis synergistic oxidation therapy.

Protein-based nanocarriers have rapidly appeared as charming drug delivery vehicles due to their high structural stability, excellent biocompatibility, fantastic biodegradability and easy tailorability [36-39]. Ferritin, an iron storage and transport protein, which could be specifically recognized by transferrin receptor 1 (TfR1) overexpressed cancer cells, has been investigated as a novel type of tumor-targeting 
platform [40, 41]. In addition, ferritin can release stored iron to induce ferroptosis by intracellular autophagic degradation [42-45]. Therefore, the intrinsic natural iron storage and tumor specific recognition features of ferritin make it more outstanding than inorganic or MOF materials in ferroptosis treatment [46]. Meanwhile, SRF, a multikinase inhibitor, is usually used as a first-line drug in clinical therapy for liver cancer but still suffers from drug resistances and serious adverse side effects, leading to unsatisfactory clinical outcomes [47-49]. Recent studies have revealed that SRF could indirectly increase LPO accumulation for ferroptosis by depleting GSH/GPX4 $[23,28,29]$, which could be combined with PDT to further boost ROS accumulation and conquer the poor efficacy of mono-therapy. On the other hand, the significantly anabatic oxygen consumption during PDT can be harnessed by hypoxiaresponsive drug vehicles to achieve on-demand drug release, avoiding the insufficient difference between normal tissues and tumor tissues [50-54]. Herein, a novel protein-based nanoreactor BCFe@SRF was developed by covalently crosslinking chlorin e6 (Ce6, a typical PS) [8] conjugated bovine serum albumin (BSA) and ferritin by hypoxia-responsive unit azobenzene (Azo), together with the SRF encapsulated inside the protein shell. The designed nanoplatform exhibited tremendous antitumor effects through multiple cascaded pathways for amplifying oxidative damage, and such a highly efficient and extremely low-toxic system is believed as a very promising candidate for future clinical application of synergistic PDT and ferroptosis therapy.

\section{Materials And Methods}

\section{Materials}

The source and purity of the involved reagents (used as received) are presented in the supporting information. All the reagents if not specified were analytically pure.

\section{Synthesis and characterization of BCFe@SRF nanosystems}

The synthesis procedures of the designed nanosystem were described step by step in the supporting information, adopting two simple steps according to previous reports with minute modifications $[28,55]$. $\mathrm{BC}$ and $\mathrm{BCFe}$ were also fabricated as control using similar approaches except the addition of ferritin and SRF (BC: without ferritin and SRF; BCFe: without SRF). In this work, the final concentration of samples applied for the following experiments was measured by Ce6 concentration. The characterization approaches of the physicochemical properties, photornetrics, drug release profile and iron cycle analysis of the products were clearly elaborated in the supporting information.

In vitro experiments

The murine hepatoma cell line hepa 1-6 and the mouse embryonic fibroblast cell line NIH 3T3 were utilized in this study to analyze the in vitro performance of BCFe@SRF. The cultured conditions of cells were according to our previous reports [7]. Notably, to imitate tumor hypoxia environment, the cells were placed in an anaerobic incubator with $2.5 \% \mathrm{O}_{2}$ when co-incubated with the samples. If not specially mentioned, different samples were given at an equivalent dosage of $1 \mu \mathrm{M}$ Ce6. In the in vitro assays, a 
$670 \mathrm{~nm}$ laser with power intensity of $50 \mathrm{~mW} \cdot \mathrm{cm}^{-2}(5 \mathrm{~min})$ was utilized as PDT exciting light. To analyze the PDT effects in hypoxic environment, the cells were coated with an oil layer when exposed to laser irradiation. After laser irradiation, the cells were incubated for another $24 \mathrm{~h}$ and then examined by the indicated methods if not specified. The in vitro toxicity[56], intracellular localization [8], PDT outcomes [8], synergistic PDT and ferroptosis outcomes and the mechanism of anticancer effects $[28,29]$ were evaluated using previously reported methods, which were detailedly introduced in the supporting information.

\section{In vivo experiments}

Male BALB/c-nude mice (3-5 weeks) purchased from Wushi Laboratory Animal Co. Ltd. (China) were employed in the in vivo assays. All the samples (at an equivalent dosage of $4 \mathrm{mg} \cdot \mathrm{kg}^{-1} \mathrm{Ce} 6$ ) were intravenously injected into the mice. After $6 \mathrm{~h}$ of injection, the laser irradiation step was started to perform. A $670 \mathrm{~nm}$ laser at a power intensity of $0.4 \mathrm{~W} \cdot \mathrm{cm}^{-2}(5 \mathrm{~min})$ was employed to operate the PDT procedures at animal level. The tumor volume and mouse body weight were recorded during the indicated treatment. The histopathology, immune-histochemical analysis and protein expression of the treated tumors were also analyzed to examine the antitumor effects of BCFe@SRF. The detailed procedures were presented point by point in the supporting information.

\section{Results And Discussion}

\section{Synthesis and characterization of BCFe@SRF}

In this work, a hypoxia-responsive protein-based nanoreactor with high tumor specificity and biocompatibility is proposed for amplified oxidation cancer therapy. Four key components are involved in the designed nanoplatforms: (1) Ce6-conjugated BSA (BSA-Ce6), widely considered as a charming protein-based platform with the huge prospect of clinical translation $[55,57,58]$, providing $670 \mathrm{~nm}$ laser activated PDT; (2) ferritin, with the ability to catalyze TME-abundant $\mathrm{H}_{2} \mathrm{O}_{2}$ into $\cdot \mathrm{OH}$ and subsequent LPO, serving as a ferroptosis inducer to amplify ROS generation when co-performed with PDT; (3) SRF, with the capacity of destroying oxidative defense systems of cancer cells by inhibiting glutamate-cystine antiport system $X_{c}{ }^{-}$and then indirectly inactivating GSH/GPX4 $[23,28,29]$, also acting as a ferroptosis promoter to further accelerate the oxidation stress; (4) Azo, a hypoxia-responsive unit that can be cleaved by azoreductase overexpressed in hypoxic condition $[52,55]$, serving as the cross-linker to improve the stability and specificity of the nanoreactor.

The tailored fabrication procedures of the BCFe@SRF were presented in Fig. 1, adopting two simple steps according to previous reports with minute modifications [28,55]. Firstly, the Ce6 was conjugated with BSA via the formation of amide bonds to obtain BSA-Ce6 (Fig. 2a). Subsequently, the hypoxia-responsive unit Azo was employed to covalently crosslink BSA-Ce6 and ferritin, simultaneously encapsulating SRF inside the particle under hydrophobic interaction. As shown in Fig. 2b, the final product Azo-crosslinked BSACe6-ferritin@SRF (BCFe@SRF) exhibited a spherical nanostructure with a diameter of $67 \pm 6 \mathrm{~nm}$. The 
dynamic light scattering (DLS) data revealed that the hydrodynamic size of BCFe@SRF was 102.6 \pm 1.3 $\mathrm{nm}$ with polydispersity index $(\mathrm{Pdl})$ of 0.28 and the zeta potential changed to $-2.7 \pm 0.6 \mathrm{mV}$ (Fig. $2 \mathrm{~d}, 2 \mathrm{e}$, Table S1), indicating the successful fabrication of BCFe@SRF. The Azo-crosslinked BSA-Ce6 (BC, without ferritin and SRF) as well as the BSA-Ce6-ferritin (BCFe, without SRF) were synthesized as control, which presented only small discrepancies of particle size and zeta potential compared with BCFe@SRF (Table S1, Fig. S1). The hydrodynamic size and Pdl of BCFe@SRF showed no great change after storing for 6 days (Fig. 2f), confirming the stable dispersity of BCFe@SRF. Next, the quantitative analysis of the functional components in BCFe@SRF was conducted by UV-Vis measurement, high performance liquid chromatography (HPLC) and inductively coupled plasma-optical emission spectrometry (ICP-OES). The loading capacities of Ce6 and SRF in BCFe@SRF were evaluated to be approximately 3.8wt\% and 2.5wt\% using the calibration curve of standards measured by UV-Vis absorption and HLPC, respectively (Fig. S2). The Fe content in BCFe@SRF was calculated to be $0.35 \mathrm{wt} \%$ detected by ICP-OES. To verify the hypoxia responsiveness of BCFe@SRF, the nanoparticle was treated with sodium dithionite $\left(\mathrm{Na}_{2} \mathrm{~S}_{2} \mathrm{O}_{4}\right)$, a chemical mimic of the hypoxia biomarker azoreductase [52], and the morphology and size distribution of degration product were evaluated by transmission electron microscopy (TEM) and DLS. The changeable structure (Fig. 2c) and decreased hydrodynamic size (Fig. 2d, 30.9 $2.1 \mathrm{~nm}$ ) of BCFe@SRF demonstrated its reductase-enzyme-triggered disassembly property, suggesting that it has enormous potential for using as hypoxia-responsive drug release vehicles.

Then, the optical properties of BCFe@SRF were analyzed. The BSA-Ce6 was firstly examined by UV-Vis absorption, and its absorption signals ascribing to Ce6 (380-440 nm and 640-700 nm) and BSA (280$295 \mathrm{~nm}$ ) were clearly shown in the spectrum (Fig. S3), confirming the connection between BSA and Ce6. As shown in Fig. 3a, a new broad absorption band ranging from $280 \mathrm{~nm}$ to $385 \mathrm{~nm}$ related to ferritin, SRF and Azo was observed in the spectrum of BCFe@SRF, which also demonstrated the successful fabrication of BCFe@SRF, consistent with the results of TEM and DLS analysis (Fig. 2b, 2d). The UV-Vis absorption spectra of $\mathrm{BC}$ and $\mathrm{BCFe}$ containing characteristic peaks of the feeding reagents also confirmed their successful preparations (Fig. 3a, Fig. S3). Meanwhile, the fluorescence of BCFe@SRF corresponding to Ce6 with a little redshift obviously decreased compared with free Ce6, which was mainly due to the aggregated fluorescence quenching effects [55], and it could be mostly recovered after BCFe@SRF degration (Fig. 3b). Then, the ROS indicator 9,10-anthracenediylbis (methylene) dimalonic acid (ABDA) was employed to evaluate the light-triggered ROS production ability of BCFe@SRF [8]. In this work, a $670 \mathrm{~nm}$ laser was utilized to trigger Ce6-based PDT as widely reported [8, 52]. Evident decrements of ABDA absorbance (350-425 nm) after laser irradiation ( $\left.50 \mathrm{~mW} \cdot \mathrm{cm}^{-2}\right)$ were observed in BCFe@SRF treated samples (Fig. 3c), whereas only slight reductions could be seen in pure BCFe@SRF or laser treated samples (Fig. S4), indicating the laser-dependent ROS generation ability of BCFe@SRF.

Next, the hypoxia-responsive drug release property of BCFe@SRF was examined also utilizing $\mathrm{Na}_{2} \mathrm{~S}_{2} \mathrm{O}_{4}$ as azoreductase simulant. The amounts of released drug from BCFe@SRF were quantitatively analyzed using the standard curve of SRF measured by HLPC (Fig. S2). As shown in Fig. 3d, no more than $15 \%$ of SRF was released from BCFe@SRF without $\mathrm{Na}_{2} \mathrm{~S}_{2} \mathrm{O}_{4}$ treatment, owing to the stability of BCFe@SRF; 
while, in the addition of $\mathrm{Na}_{2} \mathrm{~S}_{2} \mathrm{O}_{4}$, the release amount of SRF dramatically increased (more than $90 \%$ ), suggesting the hypoxia-responsive drug release potential of BCFe@SRF. Besides Ce6 and SRF, ferritin is another vital functional component in BCFe@SRF for the designed oxidation therapy. As reported, ferritin can be intracellularly degraded to release $\mathrm{Fe}^{3+}$, which can be reduced to $\mathrm{Fe}^{2+}$ by $\mathrm{GSH}[46]$, and then triggers the Fenton reaction to convert $\mathrm{H}_{2} \mathrm{O}_{2}$ into $\cdot \mathrm{OH}$, simultaneously achieving $\mathrm{Fe}^{3+}$ recycle (Fig. $3 e$ ). $\mathrm{O}$ phenanthroline was employed as a $\mathrm{Fe}^{2+}$ indicator, which could especially react with $\mathrm{Fe}^{2+}$ to form orange complexes [29]. After extracting $\mathrm{Fe}^{3+}$ from ferritin [59], the tinct of ferritin solution presented colorless, in keeping with the $\mathrm{Fe}^{3+}$ control solution (Fig. $3 f$ ). When GSH was added to transduce $\mathrm{Fe}^{3+}$ towards $\mathrm{Fe}^{2+}$, the solution turned orange. Additionally, the solution color rapidly faded when $\mathrm{H}_{2} \mathrm{O}_{2}$ was introduced, suggesting the regeneration of $\mathrm{Fe}^{3+}$. The phenomena confirmed the iron cycling of ferritin when reacted with $\mathrm{GSH}$ and $\mathrm{H}_{2} \mathrm{O}_{2}$. All the above results demonstrated that the BCFe@SRF could serve as a promising therapeutic agent for designed PDT and ferroptosis synergistic treatment.

\section{In vitro anticancer effects of the PDT and ferroptosis synergistic therapy mediated by BCFe@SRF}

To act as a biomedical reagent, very weak cytotoxicity is a basic property of nanosystem. Therefore, the in vitro toxicity of BCFe@SRF in NIH 3T3 cell line was firstly examined by CCK-8 assay. As shown in Fig. S5, it was found that BCFe@SRF exhibited limited cytotoxicity even at high dose, fitting the essential requirement for biological applications. The cellular internalization of BCFe@SRF in hepa 1-6 cell line was then analyzed by confocal laser scanning microscopy (CLSM). As shown in Fig. S6, the fluorescence signals of Ce6 within cells increased over time, indicating the cellular uptake behaviors of BCFe@SRF. Notably, when the cells were cultured with BCFe@SRF in hypoxia condition, obvious signal enhancement could be observed owing to the recovery of Ce6 fluorescence after BCFe@SRF degradation (Fig. 4a), which may results from the cleavage of Azo by highly expressed reductases in hypoxia condition $[52,55]$.

After confirming the biocompatibility and cellular uptake capacity of BCFe@SRF, its in vitro anticancer effects were then verified. In the in vitro experiments, hepa 1-6 cells were co-incubated with the samples for $4 \mathrm{~h}$ and then subsequently to $670 \mathrm{~nm}$ light $\left(50 \mathrm{~mW} \cdot \mathrm{cm}^{-2}, 5 \mathrm{~min}\right)$ in normoxic or hypoxia condition and finally examined after another $24 \mathrm{~h}$ of incubation. To select an appropriate sample concentration as the reference, $\mathrm{BC}$ (without ferritin and SRF) was firstly engaged in CCK-8 assay to evaluate the pure PDT effects in normoxic circumstance. As shown in Fig. S7, the cell viability dramatically decreased as Ce6 concentration increased upon laser irradiation, which could be reduced to less than $40 \%$ at $\mathrm{Ce} 6$ concentration of $1 \mu \mathrm{M}$. Taking both bioavailability and efficacy into consideration, the Ce6 concentration of $1 \mu \mathrm{M}$ was chosen as the reference value for the following studies. Subsequently, the therapeutic outcomes of synergistic PDT and ferroptosis therapy mediated by BCFe@SRF were analyzed by CCK-8, live/dead staining and annexin V-APC/PI apoptosis assays. In the CCK-8 assay, PBS and laser exhibited almost no cytotoxicity towards hepa 1-6 cells, and BCFe@SRF cultured cells in normoxic condition also showed high cell viability (78.9\%) (Fig. 4b). Comparing with the normoxic condition, hypoxia cause distinct cell death of BCFe@SRF treated cells (53.6\% cell viability), owing to the killing effects of released ferritin and SRF. In normoxic condition, when the BCFe@SRF treated cells were exposed to light 
irradiation, the cell viability underwent a significant decrease (36.0\%) ascribing to the PDT induced apoptosis. Additionally, it was noteworthy that the cell viability further decreased to $12.4 \%$ when the BCFe@SRF treated cells were cultured and irradiated under hypoxia condition. Although the hypoxia would compromise the PDT effects, the BCFe@SRF degradation that makes more Ce6 exposed to laser irradiation could enhance the therapeutic efficacy to a certain extent. In addition, the killing effects could be further reinforced by ferroptosis induced by the released ferritin and SRF, all these leading to the best efficacy of BCFe@SRF treated group with laser irradiation under hypoxia incubation. To reconfirm the in vitro anticancer effects of our designed BCFe@SRF, the live/dead staining assay was then performed to intuitively observe the cell status after BCFe@SRF mediated PDT and ferroptosis therapy. As shown in Fig. 4c, only a few red signals could be found in the PBS and laser groups. The BCFe@SRF induced more cell death when the cells were in hypoxic incubation. The synergistic treatment by BCFe@SRF with laser irradiation under hypoxia condition was found to be the most effective in cancer cell killing, consistent with the results of CCK-8 assay (Fig. 4b). Annexin V-APC/PI staining assay was also utilized to validate the cell viability data. As expected, a great number of cells in the synergistic treatment group (BCFe@SRF + Laser in hypoxia condition) were localized in the lower right (early apoptosis), higher right (late apoptosis) and higher left (necrosis) quadrants (Fig. 4d). The average percentage of total apoptotic and necrotic cells in the synergistic group was calculated to be $88.4 \%$, which was obviously beyond the ferroptosis therapy group (BCFe@SRF in hypoxia condition, 42.5\%) and PDT group (BCFe@SRF + Laser in normoxic condition, 69.1\%). All these results suggested that the designed BCFe@SRF exhibited great potential to be used as both PS and ferroptosis inducer with high efficiency to restrain the hypoxic tumor growth.

\section{The mechanism of in vitro anticancer effects of BCFe@SRF}

As mentioned above, we deduced that the BCFe@SRF performed the cell killing function through PDT and ferroptosis. When BCFe@SRF was exposed to hypoxic environment within cancer cells, the highlyexpressed reductases would cleave the Azo unit in BCFe@SRF to release BSA-Ce6, SRF and ferritin [52, 55], inducing ferroptosis and light-dependent PDT. Based on the established ferroptosis mechanism as illustrated in Fig. $5 \mathrm{a}, \mathrm{Fe}^{3+}$ from ferritin can deplete $\mathrm{GSH}$, transporting to $\mathrm{Fe}^{2+}$, and subsequently catalyze TME-abundant $\mathrm{H}_{2} \mathrm{O}_{2}$ into $\cdot \mathrm{OH}$, directly resulting in accelerated $\mathrm{LPO}$ generation. On the other hand, SRF can inhibit glutamate-cystine antiport system $\mathrm{X}_{\mathrm{c}}{ }^{-}$and further down-regulate GSH and GPX4 expression to destruct the cell oxidative defense system, ultimately leading to the indirect LPO promotion $[23,28,29]$. Now, the hypothetic mechanism of this BCFe@SRF mediated oxidation treatment was carefully verified.

Different samples containing free $\mathrm{Ce} 6, \mathrm{BC}$ and $\mathrm{BCFe}$ were employed as control to treat hepa 1-6 cells under normoxic or hypoxic environment and then irradiated by $670 \mathrm{~nm}$ laser $\left(50 \mathrm{~mW} \cdot \mathrm{cm}^{-2}\right)$ for $5 \mathrm{~min}$. The treated cells were analyzed after another $24 \mathrm{~h}$ of incubation. Ferrostatin-1 (Fer-1), a typical ferroptosis inhibitor [23, 29], was also used together with BCFe@SRF as control to investigate the mechanism. In the CCK8 assay (Fig. 5b), the cells in all groups without laser illumination under normoxic condition showed high viabilities $(₫ 80 \%)$. Upon light irradiation, all cells underwent extremely remarkable 
reduced survival because of the light-triggered PDT (Free Ce6: 4.9\%, BC: 41.0\%, BCFe: 31.6\%, BCFe@SRF: $31.8 \%$, BCFe@SRF + Fer-1: 40.3\%). In hypoxic incubation, because of the oxygen deficiency, the PDT effects of free Ce6 (32.1\% of cell viability) were significantly restrained, while the outcomes of BC mediated PDT showed a lesser degree of compromise (47.1\% of cell viability). This phenomenon may attribute to more Ce6 exposure to laser after Azo cleavage. Comparing the cell status of BCFe@SRF treated cells in normoxic and hypoxia condition without light illumination, owing to the ferritin and SRF induced ferroptosis, more cell death could be found in hypoxic condition group (Normoxia vs Hypoxia: $78.2 \%$ vs $52.0 \%$ ). Notably, in hypoxic incubation, the BCFe@SRF treated cells upon laser irradiation showed only $8.3 \%$ of cell viability, suggesting the further enhanced anticancer effects by co-performance of PDT and ferroptosis. Moreover, in the addition of Fer-1 that could neutralize LPO, the toxicity of BCFe@SRF was obviously inhibited, further demonstrating that the BCFe@SRF mediated cell death had a great association with LPO caused oxidative damage. After evaluating the cell killing effects, the intracellular ROS production was visibly examined by 2',7'-dichlorodihydr fluorescein diacetate (DCFH-DA) sensor which would emit green fluorescence when reacting with ROS. The cells were cultured with different samples for $4 \mathrm{~h}$ and then exposed to laser irradiation ( $670 \mathrm{~nm}$ light, $50 \mathrm{~mW} \cdot \mathrm{cm}^{-2}, 5 \mathrm{~min}$ ) in hypoxia condition. As shown in Fig. 5c, few fluorescence could be observed in pure PBS and Laser group, and the green signal intensity in BC + Laser group apparently got stronger owing to the PDT-induced ROS. The strongest fluorescence was observed in BCFe@SRF + Laser group, verifying the amplified ROS generation by simultaneous action of PDT and ferroptosis. As expected, when the Fer- 1 was added to inhibit ferroptosis-induced ROS, some degree of signal attenuations occurred. All these results explained and reconfirmed the enhanced anticancer efficacy of synergistic PDT and ferroptosis.

After verifying the synergistic mechanism, the ferroptosis induced by BCFe@SRF was now explored in detail, with BC, BCFe and BCFe@SRF + Fer-1 as control. As reported, LPO generation, GSH depletion and GPX4 inactivation were considered as three symbolic events during ferroptosis [11, 27-29]. In all the following mechanism study assays, the cells were co-incubated with different samples in hypoxic environment without laser irradiation. Firstly, the BODIPY581/591-C11 (a LPO sensor [29]) was used to examine the in vitro LPO generation. As presented in the CLSM images (Fig. 5d), owing to the contribution of ferritin-supplied LPO, the green fluorescence intensity in the cell membrane (representing LPO) in BCFe group was stronger than that in PBS or BC group. The signals were further heightened by the addition of SRF in the BCFe@SRF group, which could be greatly restrained by Fer-1, reconfirming the cytotoxicity of BCFe@SRF involves the LPO-induced ferroptosis. The quantitative analysis of produced LPO was examined by flow cytometry (Fig. 5e, 5f). The strongest mean fluorescence intensity (MFI) of LPO presented in BCFe@SRF group, reaching respectively 2.4-fold and 13.8-fold MFI enhancement in comparing with BCFe and BCFe@SRF + Fer-1 groups, which exhibited excellent consistency with the CLSM visualization (Fig. 5d). Subsequently, intracellular GSH and GPX4 were respectively evaluated by Reduced GSH Assay Kit and western blot analysis. When the cells were treated with BCFe@SRF, the GSH and GPX4 expression remarkably downgraded (Fig. 5g, 5h), implying the designed BCFe@SRF indeed destroyed the intracellular antioxidative defense system of the cancer cells. All these results verified the cytotoxicity of BCFe@SRF in hypoxia condition were mightily associated with ferroptosis. 
In vivo anticancer effects of the PDT and ferroptosis synergistic therapy mediated by BCFe@SRF

Encouraged by the in vitro results, we next investigated the in vivo performance of our designed BCFe@SRF. First of all, the tumor bearing mouse model was established and the biodistribution of BCFe@SRF after intravenous injection was detected by tracking the Ce6 fluorescence using in vivo imaging system (IVIS). As revealed in the Fig. 6a, the tumor-site Ce6 fluorescence gradually increased along with time and actually peaked its intensity by $6 \mathrm{~h}$, which demonstrated the outstanding tumor accumulation capacity of BCFe@SRF. The tumor targeting ability of such a protein-based nanoparticle mainly attributed to the passive targeting property well-known as enhanced permeation and retention (EPR) effects together with the interactions between certain proteins (albumin and ferritin) and specific receptors overexpressed on tumor cells [36-39]. Based on the IVIS observation, the mouse was sacrificed after $6 \mathrm{~h}$ of BCFe@SRF injection and the harvested tumor as well as major organs were then analyzed by ex vivo fluorescence imaging. It could be observed that the highest Ce6 fluorescent signals were found in the isolated tumor (Fig. 6b, 6c), which was concordant with the living imaging visualization (Fig. 6a). According to these results, the time point $6 \mathrm{~h}$ after injection was selected to conduct in vivo PDT procedure.

Thereafter, the in vivo antitumor effects of BCFe@SRF mediated PDT and ferroptosis synergistic therapy were investigated. The mice were randomly divided into five groups including PBS (as control), PBS + Laser, BC + Laser (pure PDT), BCFe@SRF (pure ferroptosis) and BCFe@SRF + Laser (synergistic PDT and ferroptosis therapy) groups. As illustrated in the Fig. $6 \mathrm{~d}$, the mice were injected with different samples at 0 day and then the mice in laser-involved groups were exposed to laser irradiation after $6 \mathrm{~h}$ of injection ( $670 \mathrm{~nm}$ light, $0.4 \mathrm{~W} \cdot \mathrm{cm}^{-2}, 5 \mathrm{~min}$ ). The relative tumor volume and mouse body weight were recorded during another 16 days of monitoring. All the mice were sacrificed after treatment and the excised tumors were photographed and weighed to evaluate the therapeutic effects. As shown in Fig. 6e-6g, the tumors in single PDT (BC + Laser) or ferroptosis therapy (BCFe@SRF) group showed a certain degree of growth suppression in comparison with those in PBS or PBS + Laser group. However, no remarkable tumor inhibitions in these two groups occurred, which may result from the insufficient ROS supply of these two single modality alone. In addition, it revealed obviously outstanding therapeutic outcomes when coperformed PDT and ferroptosis therapy (BCFe@SRF + Laser group), the tumor growth restrained more effectively, mainly benefiting from the amplified oxidative damage by accelerating ROS generation as well as promoting antioxidative component depletion as confirmed above (Fig. 5).

To further investigate the synergistic antitumor mechanism, one tumor-bearing mouse in each group was sacrificed after $24 \mathrm{~h}$ of the indicated laser irradiation procedures. The treated tumors were analyzed by H\&E and Ki67 immuno-histochemical staining assays to assess the histological changes. Consistent with the tumor growth evaluation results, the most severe cell morphologic changes (cytoplasm leakages and nucleus shrinkages) and the weakest cell proliferation signals (brown granules in the cell nucleus) could be observed in the synergistic therapy (BCFe@SRF + Laser) group (Fig. 6h, 6i), reconfirming the prominently preferable antitumor efficacy of our designed systems. The GPX4 expression of the harvested tumor was then evaluated by immuno-histochemical analysis. As expected, the GPX4 down- 
regulation occurred in the treatment groups, greatest degree of GPX4 reduction in BCFe@SRF + Laser group, indicating the compromised antioxidation ability of tumor after the indicated treatments.

Then, the potential systemic toxicity of BCFe@SRF was analyzed. First of all, during the therapeutic period, there was no apparent body weight change of the mice in all groups (Fig. S8), implying the biosafety of the designed BCFe@SRF. To further verify the biocompatibility of BCFe@SRF, the major organs of one mouse in each group were collected at the end of the indicated treatment. H\&E immunohistochemical staining assay were performed to assess the histopathological changes of the main organs, and the results presented in Fig. S9 turned out to be that no significant physiological abnormalities appeared in all groups, reconfirming the biological security of the BCFe@SRF.

\section{Conclusions}

In summary, a novel protein-based nanoreactor BCFe@SRF with high tumor specificity and biocompatibility was developed by covalently crosslinking BSA-Ce6 and ferritin by hypoxia-responsive unit Azo, with the SRF encapsulated inside the protein shell. The as-designed BCFe@SRF not only exhibited outstanding dispersity and stability but also could respond to hypoxic TME to release the functional components, Ce6 for laser-triggered ${ }^{1} \mathrm{O}_{2}$ generation by PDT action and ferritin for ironcatalyzed $\mathrm{H}_{2} \mathrm{O}_{2}$ into $\cdot \mathrm{OH}$ and then LPO by ferroptosis effect. Meanwhile, together with the directly amplified ROS production, the LPO accumulation could be indirectly promoted by destroying the antioxidative defense system of tumor cells through SRF-induced GSH and GPX4 depletion, which could further increase the oxidative stress within tumor sites. With excellent tumor accumulating capacity and high specificity, BCFe@SRF presented remarkable therapeutic outcomes both in vitro and in vivo. Therefore, the BCFe@SRF, integrating PDT and ferroptosis into one nanosystem with hypoxia responsiveness, offers a powerful strategy through multiple ROS enlargement cascaded pathways for future tumor-specific oxidation treatment.

\section{Declarations}

\section{Supporting Information}

Supporting Information is available from online or from the author.

\section{Ethics approval and consent to participate}

All animal experiments were operated strictly according to the "National animal management regulations of China" and the guidelines approved by the Animal Ethics Committee of Mengchao Hepatobiliary Hospital of Fujian Medical University.

\section{Consent for publication}

All authors agree to be published. 
Availability of data and materials

The datasets used during the present study are available from the corresponding author upon reasonable request.

\section{Competing interests}

There are no competing interests to declare.

\section{Funding}

This work is supported by the Natural Science Foundation of China (Grant No. 81871483); The Natural Science Foundation of Fujian Province of China (Grant No. 2020J02010); The Guiding Project of Fujian Provincial Department of Science \& Technology (Grant No. 2019Y0013); The Joint Funds for the Innovation of Science and Technology, Fujian Province (Grant No. 2018Y9119, 2019 Y9046 and 2019Y9108); The Scientific Foundation of Fuzhou Municipal Health Commission (Grant No. 2020-S-wt7 and 2020-S-wp6).

\section{Authors' contributions}

Xiaoyan Wang: Methodology, Investigation, Formal analysis, Writing-Original draft.

Ming Wu: Conceptualization and Supervision.

Xiaolong Zhang: Conceptualization and Supervision.

Feida Li: Investigation and Validation.

Yongyi Zeng: Supervision and Reviewing.

Xinyi Lin: Methodology, Supervision, Data curation, Reviewing and Editing.

Xiaolong Liu: Conceptualization, Supervision and Editing.

Jingfeng Liu: Supervision and Reviewing.

\section{Acknowledgements}

The authors want to thank Yang Li and Peiyuan Wang from Fujian Institute of Research on the Structure of Matter for technical support.

\section{References}

1. Tang ZM, Liu YY, Ni DL, Zhou JJ, Zhang M, Zhao PR, Lv B, Wang H, Jin DY, Bu WB. Biodegradable nanoprodrugs: "Delivering" ROS to cancer cells for molecular dynamic therapy. Adv Mater. 
2019:e1904011.

2. Jasim KA, Gesquiere AJ. Ultrastable and biofunctionalizable conjugated polymer nanoparticles with encapsulated iron for ferroptosis assisted chemodynamic therapy. Mol Pharm. 2019;16:4852-66.

3. Yang B, Ding L, Yao H, Chen Y, Shi J. A metal-organic framework (MOF) fenton nanoagent-enabled nanocatalytic cancer therapy in synergy with autophagy inhibition. Adv Mater. 2020;32:e1907152.

4. Wu M, Ding Y, Li L. Recent progress in the augmentation of reactive species with nanoplatforms for cancer therapy. Nanoscale. 2019;11:19658-83.

5. Wei Z, Liang P, Xie J, Song C, Tang C, Wang Y, Yin X, Cai Y, Han W, Dong X. Carrier-free nanointegrated strategy for synergetic cancer anti-angiogenic therapy and phototherapy. Chem Sci. 2019;10:2778-84.

6. Yang H, Liu R, Xu Y, Qian L, Dai Z. Photosensitizer nanoparticles boost photodynamic therapy for pancreatic cancer treatment. Nano-Micro Letters. 2021;13:1-16.

7. Ding L, Lin X, Lin Z, Wu Y, Liu X, Liu J, Wu M, Zhang X, Zeng Y. Cancer cell-targeted photosensitizer and therapeutic protein co-delivery nanoplatform based on a metal-organic framework for enhanced synergistic photodynamic and protein therapy. ACS Appl Mater Interfaces. 2020;12:36906-16.

8. Lin X, Wu M, Li M, Cai Z, Sun H, Tan X, Li J, Zeng Y, Liu X, Liu J. Photo-responsive hollow silica nanoparticles for light-triggered genetic and photodynamic synergistic therapy. Acta Biomater. 2018;76:178-92.

9. Lo PC, Rodriguez-Morgade MS, Pandey RK, Ng DKP, Torres T, Dumoulin F. The unique features and promises of phthalocyanines as advanced photosensitisers for photodynamic therapy of cancer. Chem Soc Rev. 2020;49:1041-56.

10. Gao J, Zhou H, Zhao Y, Lu L, Zhang J, Cheng W, Song X, Zheng Y, Chen C, Tang J. Time-course effect of ultrasmall superparamagnetic iron oxide nanoparticles on intracellular iron metabolism and ferroptosis activation. Nanotoxicology. 2021:1-14.

11. Meng X, Deng J, Liu F, Guo T, Liu M, Dai P, Fan A, Wang Z, Zhao Y. Triggered all-active metal organic framework: ferroptosis machinery contributes to the apoptotic photodynamic antitumor therapy. Nano Lett. 2019;19:7866-76.

12. Hu JJ, Chen Y, Li ZH, Peng SY, Sun Y, Zhang XZ. Augment of oxidative damage with enhanced photodynamic process and MTH1 inhibition for tumor therapy. Nano Lett. 2019;19:5568-76.

13. Tang Z, Liu Y, He M, Bu W. Chemodynamic therapy: Tumour microenvironment-mediated fenton and fenton-like reactions. Angew Chem Int Ed Engl. 2019;58:946-56.

14. Hwang E, Jung HS. Metal-organic complex-based chemodynamic therapy agents for cancer therapy. Chem Commun. 2020;56:8332-41.

15. Lin H, Chen Y, Shi J. Nanoparticle-triggered in situ catalytic chemical reactions for tumour-specific therapy. Chem Soc Rev. 2018;47:1938-58.

16. He Y, Hua Liu S, Yin J, Yoon J. Sonodynamic and chemodynamic therapy based on organic/organometallic sensitizers. Coord Chem Rev. 2021;429:213610. 
17. Hao YN, Zhang WX, Gao YR, Wei YN, Shu Y, Wang JH. State-of-the-art advances of copper-based nanostructures in the enhancement of chemodynamic therapy. J Mater Chem B. 2021;9:250-66.

18. Wang X, Zhong X, Liu Z, Cheng L. Recent progress of chemodynamic therapy-induced combination cancer therapy. Nano Today. 2020;35:100946.

19. Wang W, Jin Y, Xu Z, Liu X, Bajwa SZ, Khan WS, Yu H. Stimuli-activatable nanomedicines for chemodynamic therapy of cancer. Wiley Interdiscip Rev Nanomed Nanobiotechnol. 2020;12:e1614.

20. Yang B, Dai Z, Zhang G, Hu Z, Yao X, Wang S, Liu Q, Zheng X. Ultrasmall ternary FePtMn nanocrystals with acidity-triggered dual-ions release and hypoxia relief for multimodal synergistic chemodynamic/photodynamic/photothermal cancer therapy. Adv Healthc Mater. 2020;9:e1901634.

21. Liang H, Guo J, Shi Y, Zhao G, Sun S, Sun X. Porous yolk-shell Fe/Fe304 nanoparticles with controlled exposure of highly active Fe(0) for cancer therapy. Biomaterials. 2021;268:120530.

22. Wang $B$, Zhang $X$, Wang Z, Shi D. Ferroptotic nanomaterials enhance cancer therapy via boosting Fenton-reaction. J Drug Deliv Sci Technol. 2020;59:101883.

23. Shen Z, Song J, Yung BC, Zhou Z, Wu A, Chen X. Emerging strategies of cancer therapy based on ferroptosis. Adv Mater. 2018;30:e1704007.

24. Shen Z, Liu T, Li Y, Lau J, Yang Z, Fan W, Zhou Z, Shi C, Ke C, Bregadze VI, Mandal SK, Liu Y, Li Z, Xue T, Zhu G, Munasinghe J, Niu G, Wu A, Chen X. Fenton-reaction-acceleratable magnetic nanoparticles for ferroptosis therapy of orthotopic brain tumors. ACS Nano. 2018;12:11355-65.

25. Jiang Q, Wang K, Zhang X, Ouyang B, Liu H, Pang Z, Yang W. Platelet membrane-camouflaged magnetic nanoparticles for ferroptosis-enhanced cancer immunotherapy. Small. 2020;16:e2001704.

26. Chen Q, Ma X, Xie L, Chen W, Xu Z, Song E, Zhu X, Song Y. Iron-based nanoparticles for MR imagingguided ferroptosis in combination with photodynamic therapy to enhance cancer treatment. Nanoscale. 2021;13:4855-70.

27. Zhu T, Shi L, Yu C, Dong Y, Qiu F, Shen L, Qian Q, Zhou G, Zhu X. Ferroptosis promotes photodynamic therapy: supramolecular photosensitizer-inducer nanodrug for enhanced cancer treatment. Theranostics. 2019;9:3293-307.

28. Xu T, Ma Y, Yuan Q, Hu H, Hu X, Qian Z, Rolle JK, Gu Y, Li S. Enhanced ferroptosis by oxygen-boosted phototherapy based on a 2-in-1 nanoplatform of ferrous hemoglobin for tumor synergistic therapy. ACS Nano. 2020;14:3414-25.

29. Liu T, Liu W, Zhang M, Yu W, Gao F, Li C, Wang SB, Feng J, Zhang XZ. Ferrous-supply-regeneration nanoengineering for cancer-cell-specific ferroptosis in combination with imaging-guided photodynamic therapy. ACS Nano. 2018;12:12181-92.

30. Cheng YJ, Hu JJ, Qin SY, Zhang AQ, Zhang XZ. Recent advances in functional mesoporous silicabased nanoplatforms for combinational photo-chemotherapy of cancer. Biomaterials. 2020;232:119738.

31. Lin X, Wang X, Gu Q, Lei D, Liu X, Yao C. Emerging nanotechnological strategies to reshape tumor microenvironment for enhanced therapeutic outcomes of cancer immunotherapy. Biomed Mater. 2021;16:042001. 
32. Wang X, Xuan Z, Zhu X, Sun H, Li J, Xie Z. Near-infrared photoresponsive drug delivery nanosystems for cancer photo-chemotherapy. J Nanobiotechnology. 2020;18:108.

33. Zhao P, Tang Z, Chen X, He Z, He X, Zhang M, Liu Y, Ren D, Zhao K, Bu W. Ferrous-cysteinephosphotungstate nanoagent with neutral $\mathrm{pH}$ fenton reaction activity for enhanced cancer chemodynamic therapy. Mater Horiz. 2019;6:369-74.

34. Biswas C, Zhang Y, DeCastro R, Guo H, Nakamura T, Kataoka H, Nabeshima K. The human tumor cellderived collagenase stimulatory factor (renamed EMMPRIN) is a member of the immunoglobulin superfamily. Cancer Res. 1995;55:434-9.

35. Roomi MW, Monterrey JC, Kalinovsky T, Rath M, Niedzwiecki A. Distinct patterns of matrix metalloproteinase-2 and - 9 expression in normal human cell lines. Oncol Rep. 2009;21:821-6.

36. Lee EJ, Lee NK, Kim IS. Bioengineered protein-based nanocage for drug delivery. Adv Drug Deliv Rev. 2016;106:157-71.

37. Lohcharoenkal W, Wang L, Chen YC, Rojanasakul Y. Protein nanoparticles as drug delivery carriers for cancer therapy. Biomed Res Int. 2014;2014:180549.

38. Sandra F, Khaliq NU, Sunna A, Care A. Developing protein-based nanoparticles as versatile delivery systems for cancer therapy and imaging. Nanomaterials. 2019;9:1329.

39. Gaber M, Medhat W, Hany M, Saher N, Fang JY, Elzoghby A. Protein-lipid nanohybrids as emerging platforms for drug and gene delivery: Challenges and outcomes. J Control Release. 2017;254:75-91.

40. Truffi M, Fiandra L, Sorrentino L, Monieri M, Corsi F, Mazzucchelli S. Ferritin nanocages: A biological platform for drug delivery, imaging and theranostics in cancer. Pharmacol Res. 2016;107:57-65.

41. Liang M, Fan K, Zhou M, Duan D, Zheng J, Yang D, Feng J, Yan X. H-ferritin-nanocaged doxorubicin nanoparticles specifically target and kill tumors with a single-dose injection. Proc Natl Acad Sci. 2014;111:14900-5.

42. Hou W, Xie Y, Song X, Sun X, Lotze MT, Zeh HJ 3rd, Kang R, Tang D. Autophagy promotes ferroptosis by degradation of ferritin. Autophagy. 2016;12:1425-8.

43. Gao M, Monian P, Pan Q, Zhang W, Xiang J, Jiang X. Ferroptosis is an autophagic cell death process. Cell Res. 2016;26:1021-32.

44. Yang RZ, Xu WN, Zheng HL, Zheng XF, Li B, Jiang LS, Jiang SD. Involvement of oxidative stressinduced annulus fibrosus cell and nucleus pulposus cell ferroptosis in intervertebral disc degeneration pathogenesis. J Cell Physiol. 2021;236:2725-39.

45. Park E, Chung SW. ROS-mediated autophagy increases intracellular iron levels and ferroptosis by ferritin and transferrin receptor regulation. Cell Death Dis. 2019;10:822.

46. Li Y, Wang X, Yan J, Liu Y, Yang R, Pan D, Wang L, Xu Y, Li X, Yang M. Nanoparticle ferritin-bound erastin and rapamycin: A nanodrug combining autophagy and ferroptosis for anticancer therapy. Biomater Sci. 2019;7:3779-87.

47. Zhu YJ, Zheng B, Wang HY, Chen L. New knowledge of the mechanisms of sorafenib resistance in liver cancer. Acta Pharmacol Sin. 2017;38:614-22. 
48. Wilhelm S, Carter C, Lynch M, Lowinger T, Dumas J, Smith RA, Schwartz B, Simantov R, Kelley S. Discovery and development of sorafenib: a multikinase inhibitor for treating cancer. Nat Rev Drug Discov. 2006;5:835-44.

49. Llovet JM, Ricci S, Mazzaferro V, Hilgard P, Gane E, Blanc J-F, De Oliveira AC, Santoro A, Raoul J-L, Forner A. Sorafenib in advanced hepatocellular carcinoma. New Engl J Med. 2008;359:378-90.

50. Li J, Meng X, Deng J, Lu D, Zhang X, Chen Y, Zhu J, Fan A, Ding D, Kong D, Wang Z, Zhao Y. Multifunctional micelles dually responsive to hypoxia and singlet oxygen: enhanced photodynamic therapy via interactively triggered photosensitizer delivery. ACS Appl Mater Interfaces. 2018;10:17117-28.

51. Wang W, Lin L, Ma X, Wang B, Liu S, Yan X, Li S, Tian H, Yu X. Light-induced hypoxia-triggered living nanocarriers for synergistic cancer therapy. ACS Appl Mater Interfaces. 2018;10:19398-407.

52. Zhang X, Wu M, Li J, Lan S, Zeng Y, Liu X, Liu J. Light-enhanced hypoxia-response of conjugated polymer nanocarrier for successive synergistic photodynamic and chemo-therapy. ACS Appl Mater Interfaces. 2018;10:21909-19.

53. Li Z, Wu M, Bai H, Liu X, Tang G. Light-enhanced hypoxia-responsive nanoparticles for deep tumor penetration and combined chemo-photodynamic therapy. Chem Commun. 2018;54:13127-30.

54. Dai Y, Xu C, Sun X, Chen X. Nanoparticle design strategies for enhanced anticancer therapy by exploiting the tumour microenvironment. Chem Soc Rev. 2017;46:3830-52.

55. Yang G, Phua SZF, Lim WQ, Zhang R, Feng L, Liu G, Wu H, Bindra AK, Jana D, Liu Z, Zhao Y. A hypoxia-responsive albumin-based nanosystem for deep tumor penetration and excellent therapeutic efficacy. Adv Mater. 2019;31:e1901513.

56. Lin X, Wang X, Li J, Cai L, Liao F, Wu M, Zheng D, Zeng Y, Zhang Z, Liu X, Wang J, Yao C. Localized NIR-II photo-immunotherapy through the combination of photothermal ablation and in situ generated interleukin-12 cytokine for efficiently eliminating primary and abscopal tumors. Nanoscale. 2021;13:1745-58.

57. Chen Q, Liu Z. Albumin carriers for cancer theranostics: a conventional patform with new promise. Adv Mater. 2016;28:10557-66.

58. Zhang N, Zhao F, Zou Q, Li Y, Ma G, Yan X. Multitriggered tumor-responsive drug delivery vehicles based on protein and polypeptide coassembly for enhanced photodynamic tumor ablation. Small. 2016;12:5936-43.

59. La A, Nguyen T, Tran K, Sauble E, Tu D, Gonzalez A, Kidane TZ, Soriano C, Morgan J, Doan M, Tran K, Wang CY, Knutson MD, Linder MC. Mobilization of iron from ferritin: new steps and details. Metallomics. 2018;10:154-68.

\section{Figures}



O +
- $\underset{\mathrm{NHS}}{\stackrel{\text { EDC }}{\longrightarrow}}$

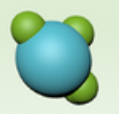
$+\quad+$

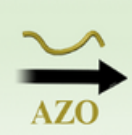
BSA
Ce6
BSA-Ce6
Ferritin
SRF
BCFe@SRF

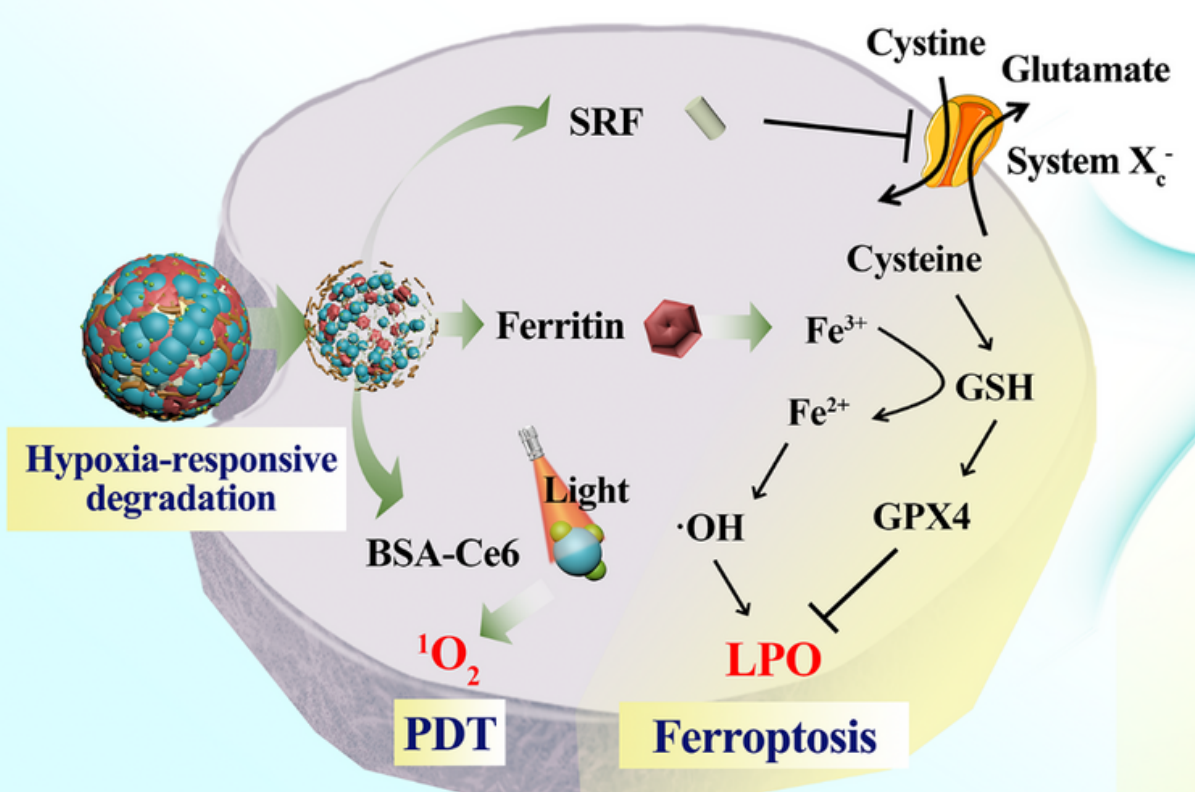

\section{Figure 1}

The fabrication procedures and antitumor mechanisms of BCFe@SRF nanoreactor for the designed synergistic PDT and ferroptosis therapy. 

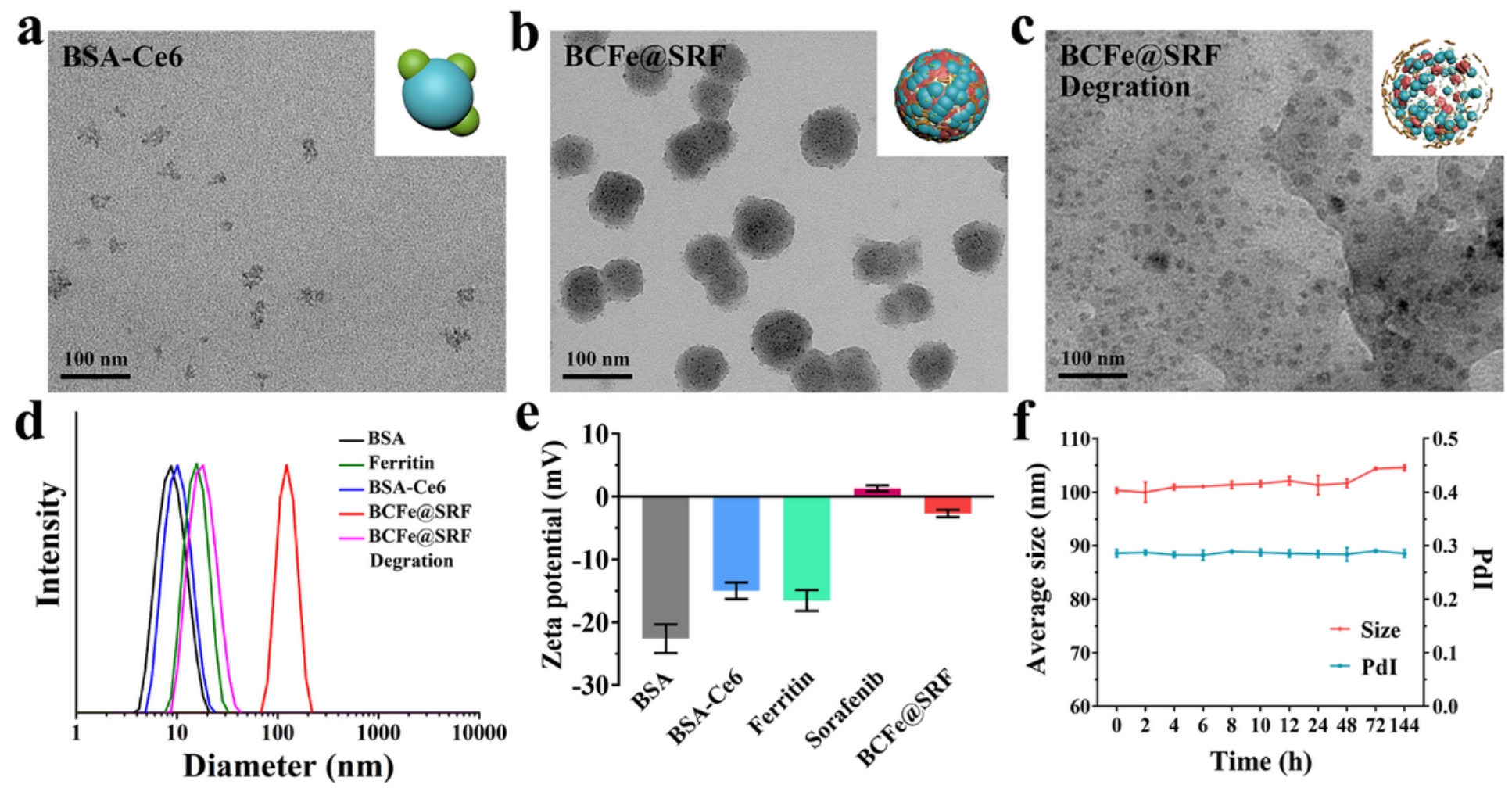

Figure 2

TEM images of (a) BSA-Ce6, (b) BCFe@SRF, and (c) BCFe@SRF degration product. (d) Size distribution of the reagents, final BCFe@SRF and BCFe@SRF degration product measured by DLS. (e) Zeta potential of the reagents and final BCFe@SRF determined by DLS. (f) Size distribution and PdI of BCFe@SRF in water after storing for 6 days measured by DLS.
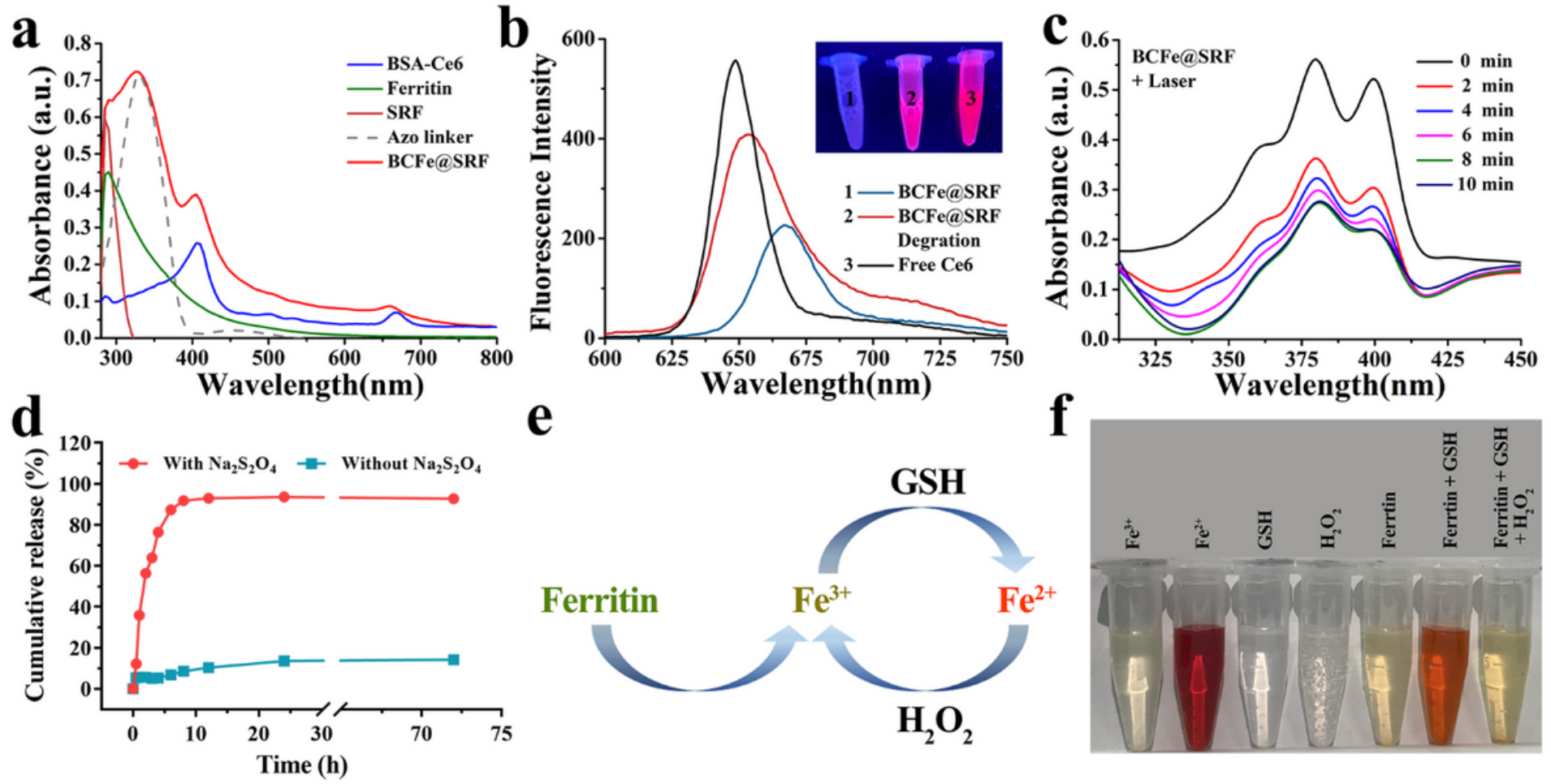


\section{Figure 3}

(a) UV-Vis absorption spectra of the feeding reagents and the final product BCFe@SRF. (b) Fluorescence emission spectra of free Ce6, BCFe@SRF and BCFe@SRF degration product excited by 404 nm laser. Insert: the corresponding fluorescence images. (c) The spectra of ABDA absorbance treated with BCFe@SRF upon different times of laser irradiation $(670 \mathrm{~nm}, 50 \mathrm{~mW} \cdot \mathrm{cm}-2)$. (d) SRF release profiles from BCFe@SRF in PBS with or without Na2S2O4 at $37^{\circ} \mathrm{C}$. (e) The schematic diagram of the iron redox cycling. (f) Observation on the iron redox cycling of ferritin.
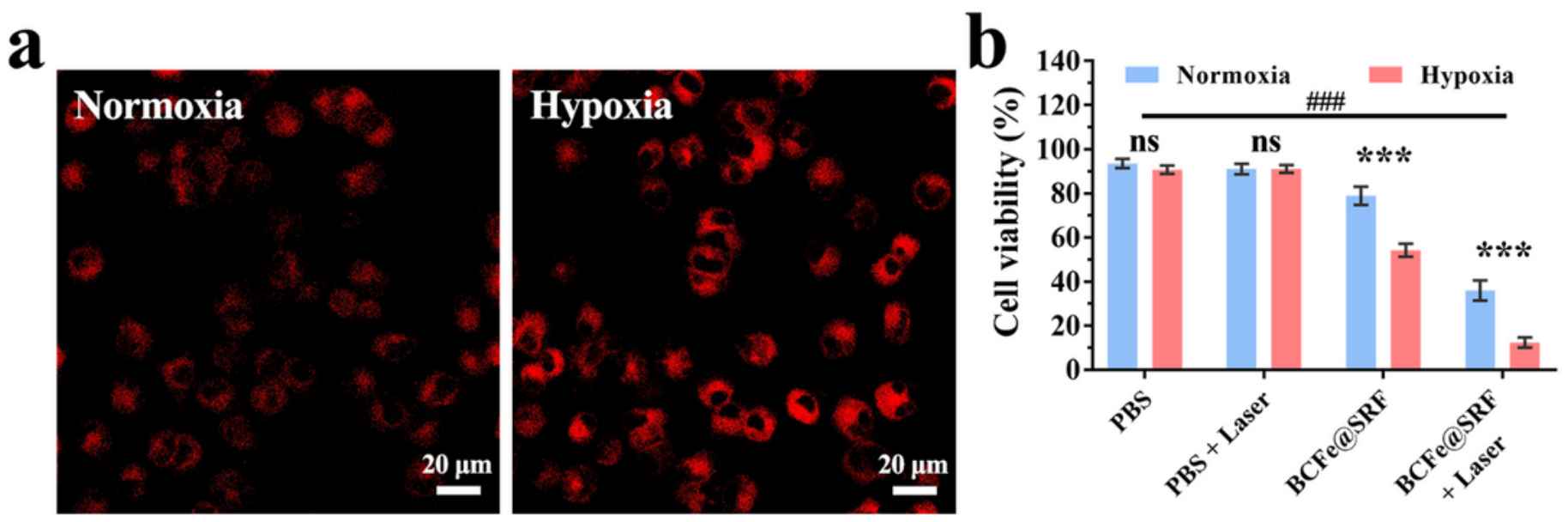

c
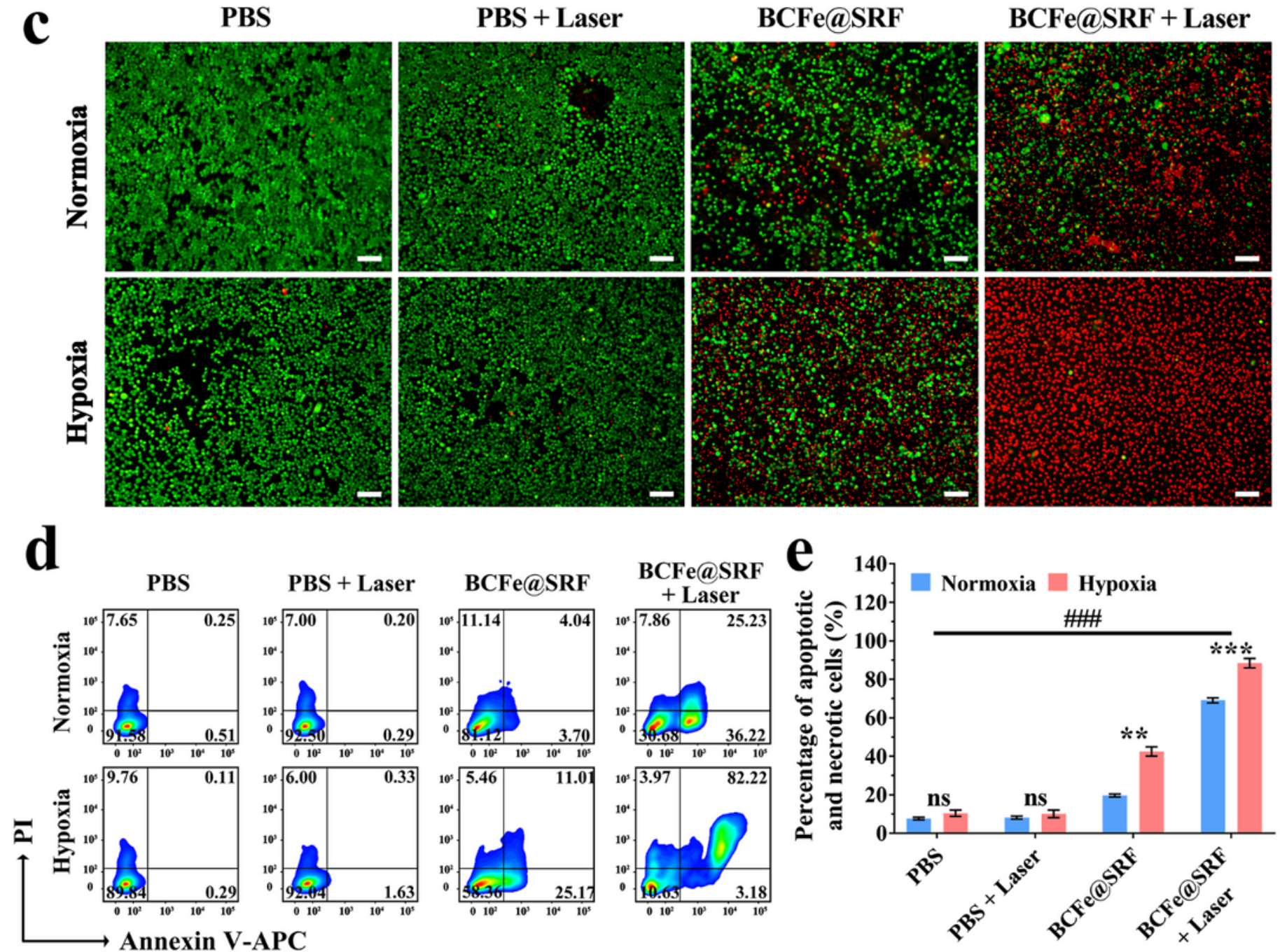


\section{Figure 4}

(a) CLSM images of hepa 1-6 cells treated with BCFe@SRF (Ce6 concentration: $1 \mu \mathrm{M}$ ) for $4 \mathrm{~h}$ in normoxic or hypoxia condition (Ce6: red, $405 \mathrm{~nm}$ laser excitation). (b) CCK-8 cell viability assay of hepa 1-6 cells treated with BCFe@SRF (Ce6 concentration: 1 $\mu \mathrm{M}$ ) mediated PDT (670 nm light, $50 \mathrm{~mW} \cdot \mathrm{cm}-2,5 \mathrm{~min}$ ) in normoxic or hypoxia condition ( ${ }^{\star} p<0.05$, ${ }^{\star \star} p<0.01$, ${ }^{\star \star \star} p<0.001$, pairwise comparison; \#p $<0.05$, \#\#p $<$ 0.01, \#\#\#p < 0.001, compared to the BCFe@SRF+Laser group in hypoxia condition, $n=5)$. (c) Live/dead staining assay (green: live cells; red: dead cells) and (d) annexin V-APC/PI apoptosis assay of hepa 1-6 cells treated with BCFe@SRF (Ce6 concentration: $1 \mu \mathrm{M}$ ) mediated PDT ( $670 \mathrm{~nm}$ light, $50 \mathrm{~mW} \cdot \mathrm{cm}-2,5 \mathrm{~min}$ ) in normoxic or hypoxia condition. (e) Percentage of apoptotic and necrotic cells in annexin V-APC/PI apoptosis assay $\left({ }^{\star} p<0.05,{ }^{*} \mathrm{p}<0.01\right.$, ${ }^{\star \star *} \mathrm{p}<0.001$, pairwise comparison; $\# p<0.05, \# \# p<0.01, \# \# \# p<$ 0.001, compared to the BCFe@SRF+Laser group in hypoxia condition, $\mathrm{n}=3$ ). 

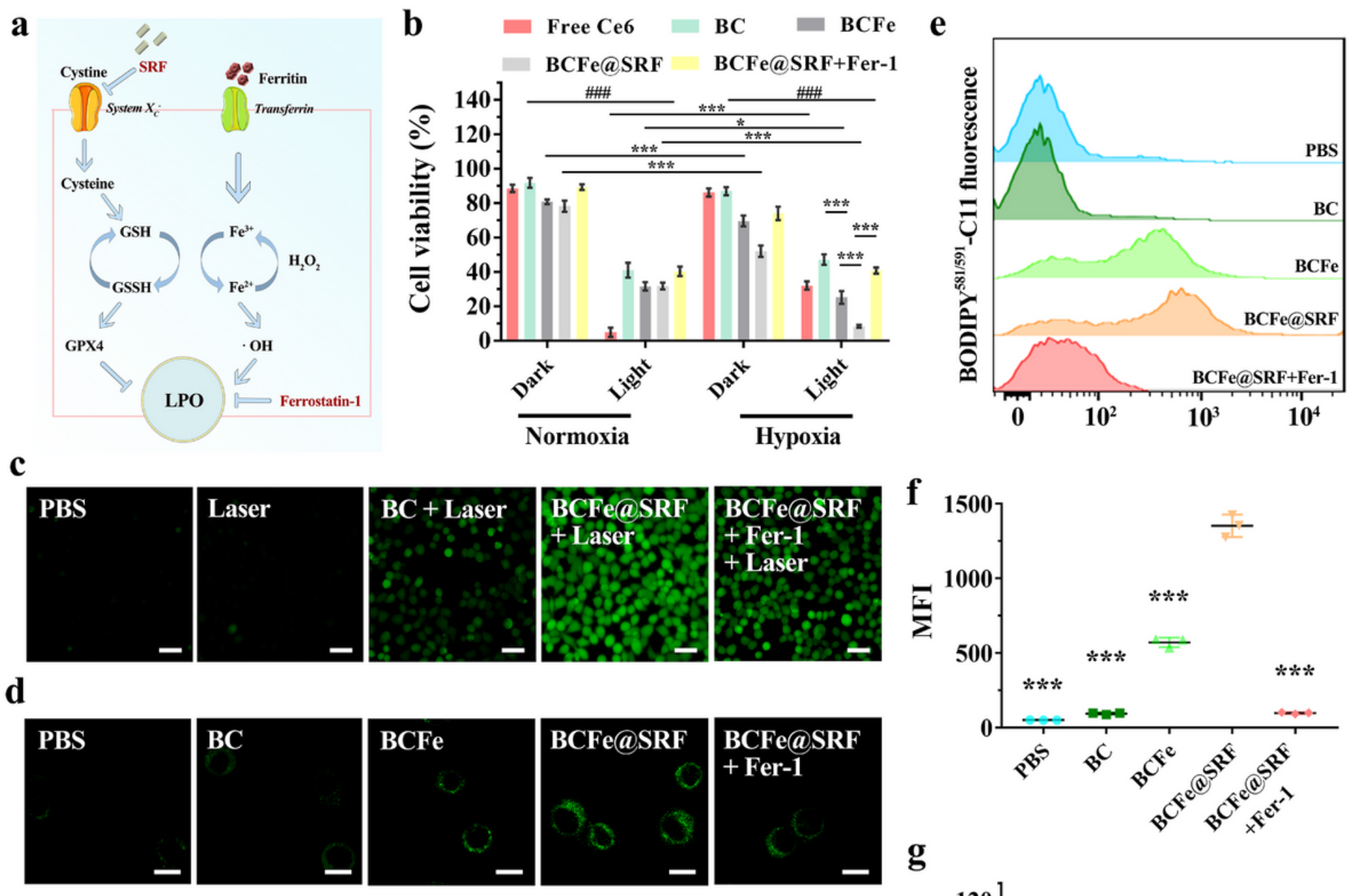

h

\section{GPX4}

Actin

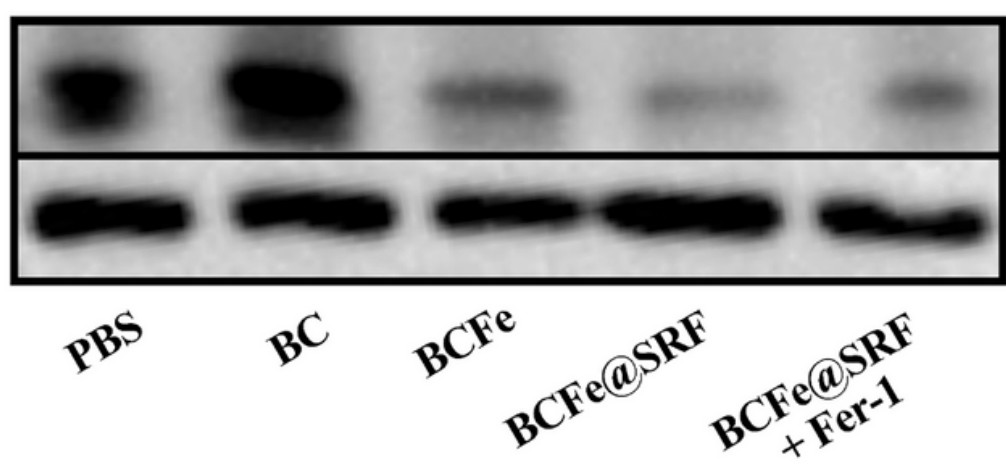

8

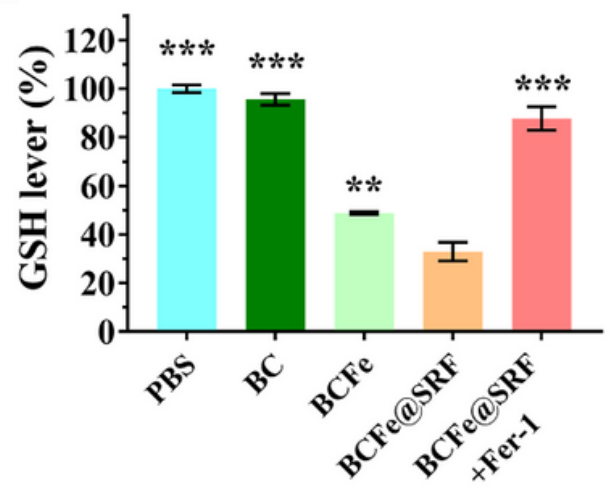

Figure 5

The mechanism of in vitro anticancer effects of BCFe@SRF. The hepa 1-6 cells were treated with different samples (Ce6 concentration: $1 \mu \mathrm{M}$ ) with or without laser irradiation ( $670 \mathrm{~nm}$ light, $50 \mathrm{~mW} \cdot \mathrm{cm}-2,5 \mathrm{~min}$ ) in normoxic or hypoxic condition. (a) The simplified mechanism of ferroptosis. (b) CCK-8 cell viability assay ( ${ }^{\star} p<0.05,{ }^{\star \star} p<0.01,{ }^{\star \star \star} p<0.001$, pairwise comparison; $\# p<0.05, \# \# p<0.01, \# \# \# p<0.001$, comparison between the same sample in normoxic and hypoxic condition, $n=5$ ). (c) Fluorescence microscopy images of hepa 1-6 cells treated with different samples and ROS indicator DCFH-DA with or without laser irradiation in hypoxic condition. (d) CLSM images of hepa 1-6 cells treated with different samples and LPO indicator BODIPY581/591-C11 in hypoxic condition. (e) The flow cytometry analysis of BODIPY581/591-C11 labeled hepa 1-6 cells treated with different samples in hypoxic incubation. (f) The 
corresponding mean fluorescence intensity (MFI) of BODIPY581/591-C11 labeled hepa 1-6 cells analyzed by flow cytometry ( ${ }^{\star} p<0.05,{ }^{*} p<0.01,{ }^{* \star *} p<0.001$, compared to the BCFe@SRF group, $\left.\mathrm{n}=3\right)$. (g) Intracellular GSH levels of the hepa 1-6 cells treated with different samples (Ce6 concentration: $1 \mu \mathrm{M}$ ) in hypoxic condition. (h) Western blot analysis of intracellular GPX4 expression of hepa 1-6 cells treated with different samples in hypoxic environment.

$\mathbf{a}$
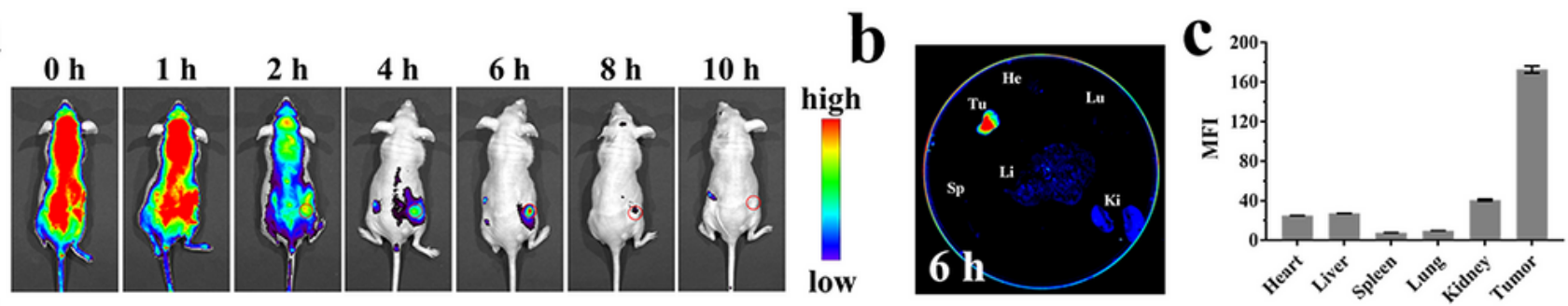

d
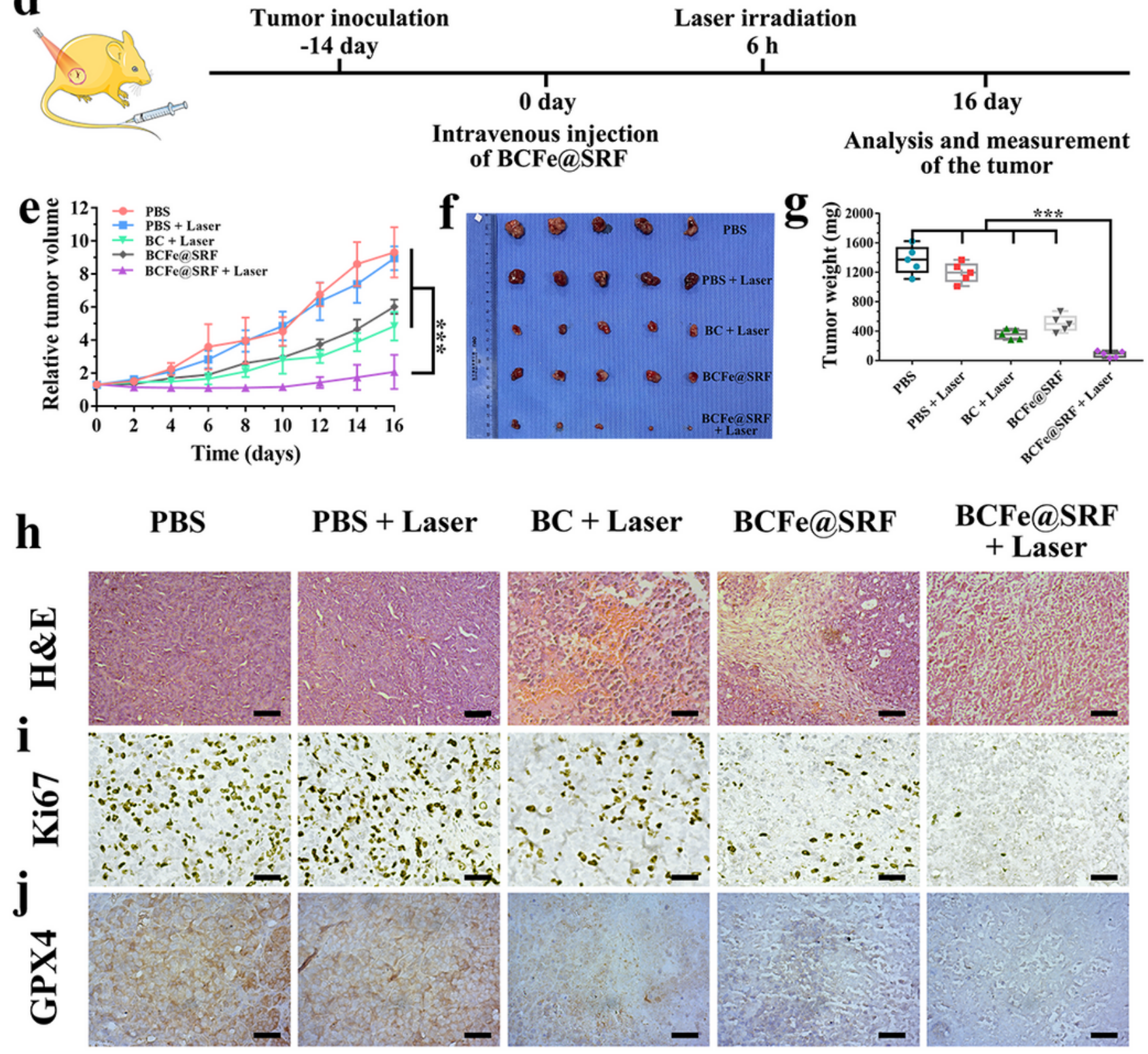

Figure 6 
In vivo performance of BCFe@SRF in hepa 1-6 tumor bearing model $\left({ }^{*} p<0.05,{ }^{*} p<0.01,{ }^{* *} p<0.001, n\right.$ $=5)$. The involved laser irradiation ( $670 \mathrm{~nm}$ light, $0.4 \mathrm{~W} \cdot \mathrm{cm}-2,5 \mathrm{~min})$ was performed after $6 \mathrm{~h}$ of sample injection. (a) In vivo fluorescence imaging of the mouse at different time points after intravenous injection of BCFe@SRF. (b) Ex vivo fluorescence imaging of the tumor and major organs harvested after 6 $\mathrm{h}$ of intravenous injection of BCFe@SRF. (c) The corresponding mean fluorescence intensity (MFI) of the harvested tumor and organs. (d) The schedule of the in vivo treatment for BCFe@SRF mediated PDT and ferroptosis synergistic therapy. (e) Time-dependent tumor growth curves. (f) The photographs and (g) average weights of the excited tumors at the end of the indicated treatment. (h) H\&E, (i) Ki67 and (j) GPX4 immuno-histochemical staining of the dissected tumor after $24 \mathrm{~h}$ of the indicated treatment. Scale bar: $50 \mu \mathrm{m}$.

\section{Supplementary Files}

This is a list of supplementary files associated with this preprint. Click to download.

- SI.docx 